\title{
Synthesis and antimicrobial activity of some pyrazoline derivatives bearing amide moiety
}

\author{
Ahmet Özdemir ${ }^{1}$
}

\begin{abstract}
In the present study, a series of pyrazoline derivatives were synthesized. The chemical structures of the compounds were elucidated by IR, ${ }^{1} \mathrm{H}-\mathrm{NMR},{ }^{13} \mathrm{C}-\mathrm{NMR}$ and FAB ${ }^{+}$ MS spectral data and elemental analyses. The synthesized compounds were screened for their antimicrobial activities. All compounds exhibited the highest antibacterial activity against $P$. aeruginosa. All compounds except compounds 1-(chloroacetyl)-3-(2-furanyl)-5-(4chlorophenyl)-2-pyrazoline and 1-(chloroacetyl)-3-(2-thienyl)-5-(3,4-methylenedioxyphenyl)2-pyrazoline are more effective than ketoconazole against $C$. albicans, whereas compounds 1-(chloroacetyl)-3-(2-furanyl)-5-(4-chlorophenyl)-2-pyrazoline, 1-(chloroacetyl)-3-(2-thienyl)5-(3,4-methylenedioxyphenyl)-2-pyrazoline and ketoconazole showed the same level of antifungal activity against $C$. albicans.
\end{abstract}

KEY WORDS: pyrazoline, amide, furan, thiophene, antimicrobial activity

\section{INTRODUCTION}

Microbial resistance to antimicrobial agents still remains a major challenge in the medicinal chemistry, as many species of bacteria and fungi have developed resistance to currently available antimicrobial drugs. Therefore, there could be a major global healthcare problem in the clinical management of life-threatening infectious diseases caused by multi-drug resistant of Gram-positive pathogens like Staphylococcus, and Gram-negative pathogens like Escherichia, and Pseudomonas strains. In order to overcome this serious problem, the search for new antimicrobial agents have gained great importance (1-3).

Medicinal chemists have carried out considerable research for novel antimicrobial agents carrying haloacetamide moiety. Chloramphenicol and analogues, which are widely used antibiotics for the treatment of systemic bacterial infections, possess dichloroacetamide group. Dichloroacetamide group is one of the functional groups that determine antibacterial activity of chlorampheni- col. Loss of this group results in significant loss of biological activity $(4,5)$.

Bioisosteric replacement approach was used for designing the compounds. The bioisosteric replacement of benzene with a heteroaromatic ring resulted in analogues maintaining the same biological activity within a series of different pharmacological agents, lending great importance to ring-equivalent bioisosteres. Furan, and thiophene, which are structurally related to benzene, are widely used as ring equivalents in drug development (6-8). $\beta$-lactam antibiotics, which possess thiophene ring as the side chain, play a leading role in the treatment of systemic bacterial infections. Tioconazole is also an example of antifungal agents bearing thiophene moiety (4). Furthermore, nitrofuran derivatives represent an important class of antimicrobial agents in medicinal chemistry (9).

Pyrazolines are widely used and studied privileged pharmacophores in medicinal chemistry
AFFILIATIONS

${ }^{1}$ Anadolu University, Faculty of Pharmacy, Deparment of Pharmaceutical Chemistry, 26470, Eskisehir, Turkey

\section{CORRESPONDENCE}

Ahmet Özdemir

E-mail:

ahmeto@anadolu.edu.tr

Received:

10.07.2013

Revision:

15.08.2013

Accepted:

16.08.2013 
due to their synthetic and biological importance. Some studies have confirmed that pyrazoline derivatives possess antimicrobial activity (10-15).

In continuation of our previous work on the synthesis and antimicrobial evaluation of pyrazoline derivatives (11-15), herein we described the discovery of 2-pyrazoline derivatives bearing three important functional moieties, namely haloacetamide, furan and thiophene and focused on their potential antimicrobial effects.

\section{MATERIALS AND METHODS Chemistry}

All reagents were used as purchased from commercial suppliers without further purification. Melting points were determined by using an Electrothermal 9100 digital melting point apparatus and were uncorrected (Electrothermal, Essex, UK). The compounds were checked for purity by TLC on silica gel 60 F254. Spectroscopic data were recorded on the following instruments: IR, Shimadzu 435 IR spectrophotometer (Shimadzu, Tokyo, Japan); ${ }^{1} \mathrm{H}-\mathrm{NMR}$, Bruker $500 \mathrm{MHz}$ NMR spectrometer (Bruker Bioscience, Billerica, MA, USA) and ${ }^{13} \mathrm{C}$ NMR, Bruker Avance II $125 \mathrm{MHz}$ NMR spectrometer (Bruker Bioscience, Billerica, MA, USA) in DMSO- $\mathrm{d}_{6}$ using TMS as internal standard; MS-FAB, VG Quattro mass spectrometer (Fisons Instruments Vertriebs GmbH, Mainz, Germany), Elemental analyses were performed on a Perkin Elmer EAL 240 elemental analyser (Perkin-Elmer, Norwalk, CT, USA).

\section{General procedure for the synthesis of the compounds 1-(2-Furanyl/thienyl)-3-aryl-2-propen-1-ones (3a-h)}

A mixture of 2-acetylfuran/thiophene (0.06 mol) (1), aromatic aldehyde $(0.06 \mathrm{~mol})(2)$, and $10 \%$ aqueous sodium hydroxide $(10 \mathrm{~mL})$ in ethanol $(30 \mathrm{~mL})$ was stirred at room temperature for about $5 \mathrm{~h}$. The resulting solid was washed, dried, and crystallized from ethanol $(16,17)$.

\section{5-Aryl-3-(2-furanyl/thienyl)-2-pyrazolines (4a-h)}

A solution of the appropriate furanyl/thienyl chalcone $(0.03$ $\mathrm{mol})$ and hydrazine hydrate (80\%) (0.06 mol) in ethanol (30 $\mathrm{mL}$ ) was refluxed for $3 \mathrm{~h}$. The reaction mixture was cooled and kept at $0^{\circ} \mathrm{C}$ overnight. The resulting solid was recrystallized from ethanol $(16,17)$.

\section{1-(Chloroacetyl)-3-(2-furanyl/thienyl)-5-aryl-2-pyrazolines (5a-h)}

5-Aryl-3-(2-furanyl/thienyl)-2-pyrazolines (0.02 mol) and triethylamine $(0.02 \mathrm{~mol})$ were dissolved in dry acetone $(30 \mathrm{~mL})$ with constant stirring. Later, the mixture was cooled in an ice bath, and chloroacetyl chloride $(0.02 \mathrm{~mol})$ was added dropwise with stirring. The reaction mixture thus obtained was further agitated for $2 \mathrm{~h}$ at room temperature. The precipitate was filtered, the solvent was evaporated to dryness under reduced pressure, and the products were recrystallized from ethanol.

\section{1-(Chloroacetyl)-3-(2-furanyl)-5-(4-methylphenyl)-2- pyrazoline (5a)}

IR (KBr) vmax $\left(\mathrm{cm}^{-1}\right): 1662(\mathrm{C}=\mathrm{O}), 1558,1488,1436(\mathrm{C}=\mathrm{N}$ and $\mathrm{C}=\mathrm{C})$.

${ }^{1} \mathrm{H}-\mathrm{NMR}\left(500 \mathrm{MHz}, \delta\right.$ ppm, DMSO- $\left.d_{6}\right)$ : $2.32(3 \mathrm{H}, \mathrm{s}), 3.04(1 \mathrm{H}, \mathrm{dd}$, $J=18.02,4.52 \mathrm{~Hz}), 3.81(1 \mathrm{H}, \mathrm{dd}, J=18.04,11.61 \mathrm{~Hz}), 4.60(1 \mathrm{H}, \mathrm{d}$, $J=13.73 \mathrm{~Hz}), 4.71(1 \mathrm{H}, \mathrm{d}, J=13.74 \mathrm{~Hz}), 5.51(1 \mathrm{H}, \mathrm{dd}, J=11.62$,
$4.51 \mathrm{~Hz}), 6.68(1 \mathrm{H}, \mathrm{t}, J=3.51,2.02 \mathrm{~Hz}), 7.04(1 \mathrm{H}, \mathrm{d}, J=3.52 \mathrm{~Hz})$, $7.09(2 \mathrm{H}, \mathrm{d}, J=8.03 \mathrm{~Hz}), 7.15(2 \mathrm{H}, \mathrm{d}, J=8.01 \mathrm{~Hz}), 7.90(1 \mathrm{H}, \mathrm{s})$.

${ }^{13} \mathrm{C}-\mathrm{NMR}\left(125 \mathrm{MHz}, \mathrm{DMSO}-d_{6}\right): 21.10\left(\mathrm{CH}_{3}\right), 42.33\left(\mathrm{CH}_{2}\right)$, $42.85\left(\mathrm{CH}_{2}\right), 59.69(\mathrm{CH}), 112.79(\mathrm{CH}), 115.46(\mathrm{CH}), 125.93$ (2CH), $129.72(2 \mathrm{CH}), 137.17(\mathrm{C}), 138.86(\mathrm{C}), 146.17(\mathrm{CH}), 146.62$ (C), $147.39(\mathrm{C}), 163.46(\mathrm{C})$.

For $\mathrm{C}_{16} \mathrm{H}_{15} \mathrm{ClN}_{2} \mathrm{O}_{2}$ calculated: $63.47 \% \mathrm{C}, 4.99 \% \mathrm{H}, 9.25 \% \mathrm{~N}$; found: $63.45 \% \mathrm{C}, 5.02 \% \mathrm{H}, 9.18 \% \mathrm{~N}$.

MS (FAB) $[\mathrm{M}+1]^{+}: \mathrm{m} / \mathrm{z} 303$

1-(Chloroacetyl)-3-(2-furanyl)-5-(3,4methylenedioxyphenyl)-2-pyrazoline (5b)

IR (KBr) vmax $\left(\mathrm{cm}^{-1}\right): 1658(\mathrm{C}=\mathrm{O}), 1564,1506,1446(\mathrm{C}=\mathrm{N}$ and $\mathrm{C}=\mathrm{C})$.

${ }^{1} \mathrm{H}-\mathrm{NMR}\left(500 \mathrm{MHz}, \delta \mathrm{ppm}, \mathrm{DMSO}-d_{6}\right): 3.06(1 \mathrm{H}, \mathrm{dd}, J=18.01$, $4.54 \mathrm{~Hz}), 3.78(1 \mathrm{H}, \mathrm{dd}, J=18.03,11.58 \mathrm{~Hz}), 4.61(1 \mathrm{H}, \mathrm{d}, J=13.74$ $\mathrm{Hz}), 4.73(1 \mathrm{H}, \mathrm{d}, J=13.71 \mathrm{~Hz}), 5.48(1 \mathrm{H}, \mathrm{dd}, J=11.57,4.53 \mathrm{~Hz})$, $6.00(2 \mathrm{H}, \mathrm{s}), 6.67-7.05(5 \mathrm{H}, \mathrm{m}), 7.93(1 \mathrm{H}, \mathrm{s})$.

${ }^{13} \mathrm{C}-\mathrm{NMR}\left(125 \mathrm{MHz}, \mathrm{DMSO}-d_{6}\right): 42.33\left(\mathrm{CH}_{2}\right), 42.94\left(\mathrm{CH}_{2}\right)$, $59.68(\mathrm{CH}), 101.52\left(\mathrm{CH}_{2}\right), 108.79(\mathrm{CH}), 112.79(\mathrm{CH}), 115.46$ $(\mathrm{CH}), 119.30(\mathrm{CH}), 135.69(\mathrm{CH}), 146.26(\mathrm{CH}), 146.45(\mathrm{C}), 146.62$ (C), 147.00 (C), 147.37 (C), 148.01 (C), 163.52 (C).

For $\mathrm{C}_{16} \mathrm{H}_{13} \mathrm{ClN}_{2} \mathrm{O}_{4}$ calculated: $57.76 \% \mathrm{C}, 3.94 \% \mathrm{H}, 8.42 \% \mathrm{~N}$; found: $57.68 \% \mathrm{C}, 3.89 \% \mathrm{H}, 8.44 \% \mathrm{~N}$.

MS (FAB) $[\mathrm{M}+1]^{+}: \mathrm{m} / \mathrm{z} 333$

\section{1-(Chloroacetyl)-3-(2-furanyl)-5-(4-methoxyphenyl)-2- pyrazoline (5c) (17)}

IR (KBr) vmax $\left(\mathrm{cm}^{-1}\right)$ : 1662 (C=O), 1610, 1512, 1463, $1440(\mathrm{C}=\mathrm{N}$ and $\mathrm{C}=\mathrm{C})$.

1H-NMR (500 MHz, $\delta$ ppm, DMSO- $\left.d_{6}\right): 3.06(1 \mathrm{H}, \mathrm{dd}, J=18.02$, $4.52 \mathrm{~Hz}), 3.73(3 \mathrm{H}, \mathrm{s}), 3.82(1 \mathrm{H}, \mathrm{dd}, J=18.03,11.48 \mathrm{~Hz}), 4.60$ $(1 \mathrm{H}, \mathrm{d}, J=13.76 \mathrm{~Hz}), 4.70(1 \mathrm{H}, \mathrm{d}, J=13.77 \mathrm{~Hz}), 5.50(1 \mathrm{H}, \mathrm{dd}, J=$ $11.48,4.52 \mathrm{~Hz}), 6.69(1 \mathrm{H}, \mathrm{t}, J=3.52,1.57 \mathrm{~Hz}), 6.89(2 \mathrm{H}, \mathrm{d}, J=$ $8.52 \mathrm{~Hz}), 7.05(1 \mathrm{H}, \mathrm{d}, J=3.02 \mathrm{~Hz}), 7.13(2 \mathrm{H}, \mathrm{d}, J=8.51 \mathrm{~Hz}), 7.93$ $(1 \mathrm{H}, \mathrm{s})$.

${ }^{13} \mathrm{C}-\mathrm{NMR}\left(125 \mathrm{MHz}, \mathrm{DMSO}-d_{6}\right): 42.28\left(\mathrm{CH}_{2}\right), 42.87\left(\mathrm{CH}_{2}\right)$, $55.58\left(\mathrm{CH}_{3}\right), 59.43(\mathrm{CH}), 112.79(\mathrm{CH}), 114.53(2 \mathrm{CH}), 115.43(\mathrm{C})$, $127.32(2 \mathrm{CH}), 128.63(\mathrm{CH}), 131.52(\mathrm{CH}), 133.89(\mathrm{C}), 152.62(\mathrm{C})$, $158.60(\mathrm{C}), 163.28(\mathrm{C})$.

For $\mathrm{C}_{16} \mathrm{H}_{15} \mathrm{ClN}_{2} \mathrm{O}_{3}$ calculated: $60.29 \% \mathrm{C}, 4.74 \% \mathrm{H}, 8.79 \% \mathrm{~N}$; found: $60.31 \% \mathrm{C}, 4.71 \% \mathrm{H}, 8.72 \% \mathrm{~N}$.

MS (FAB) $[\mathrm{M}+1]^{+}: \mathrm{m} / \mathrm{z} 319$

\section{1-(Chloroacetyl)-3-(2-furanyl)-5-(4-bromophenyl)-2- pyrazoline (5d)}

IR (KBr) vmax $\left(\mathrm{cm}^{-1}\right): 1683(\mathrm{C}=\mathrm{O}), 1564,1488,1456(\mathrm{C}=\mathrm{N}$ and $\mathrm{C}=\mathrm{C})$.

${ }^{1} \mathrm{H}-\mathrm{NMR}\left(500 \mathrm{MHz}, \delta\right.$ ppm, DMSO- $\left.d_{6}\right): 3.09$ (1H, dd, $J=18.06$, $4.74 \mathrm{~Hz}), 3.84(1 \mathrm{H}, \mathrm{dd}, J=18.04,11.72 \mathrm{~Hz}), 4.62(1 \mathrm{H}, \mathrm{d}, J=13.85$ $\mathrm{Hz}), 4.72(1 \mathrm{H}, \mathrm{d}, J=13.87 \mathrm{~Hz}), 5.54(1 \mathrm{H}, \mathrm{dd}, J=11.71,4.73 \mathrm{~Hz})$, $6.69(1 \mathrm{H}, \mathrm{t}, J=3.52,2.02 \mathrm{~Hz}), 7.05(1 \mathrm{H}, \mathrm{d}, J=3.50 \mathrm{~Hz}), 7.19(2 \mathrm{H}$, $\mathrm{d}, J=8.52 \mathrm{~Hz}), 7.54(2 \mathrm{H}, \mathrm{d}, J=8.50 \mathrm{~Hz}), 7.94(1 \mathrm{H}, \mathrm{s})$.

For $\mathrm{C}_{15} \mathrm{H}_{12} \mathrm{BrClN}_{2} \mathrm{O}_{2}$ calculated: $49.01 \% \mathrm{C}, 3.29 \% \mathrm{H}, 7.62 \% \mathrm{~N}$; found: $49.04 \% \mathrm{C}, 3.27 \% \mathrm{H}, 7.65 \% \mathrm{~N}$. 
MS (FAB) $[\mathrm{M}+1]^{+}: \mathrm{m} / \mathrm{z} 368$

\section{1-(Chloroacetyl)-3-(2-furanyl)-5-(4-chlorophenyl)-2- pyrazoline (5e)}

IR (KBr) vmax $\left(\mathrm{cm}^{-1}\right): 1677(\mathrm{C}=\mathrm{O}), 1556,1488,1427(\mathrm{C}=\mathrm{N}$ and $\mathrm{C}=\mathrm{C})$.

${ }^{1} \mathrm{H}-\mathrm{NMR}\left(500 \mathrm{MHz}, \delta\right.$ ppm, DMSO- $\left.d_{6}\right): 3.03(1 \mathrm{H}, \mathrm{dd}, J=18.08$, $4.58 \mathrm{~Hz}), 3.82(1 \mathrm{H}, \mathrm{m}), 4.57(1 \mathrm{H}, \mathrm{d}, J=13.78 \mathrm{~Hz}), 4.67(1 \mathrm{H}, \mathrm{d}, J=$ $13.79 \mathrm{~Hz}), 5.54(1 \mathrm{H}, \mathrm{dd}, J=11.68,4.58 \mathrm{~Hz}), 6.65(1 \mathrm{H}, \mathrm{t}, J=3.52$, $2.01 \mathrm{~Hz}), 7.01(1 \mathrm{H}, \mathrm{d}, J=3.50 \mathrm{~Hz}), 7.22(2 \mathrm{H}, \mathrm{d}, J=8.02 \mathrm{~Hz}), 7.39$ $(2 \mathrm{H}, \mathrm{d}, J=8.08 \mathrm{~Hz}), 7.85(1 \mathrm{H}, \mathrm{s})$.

${ }^{13} \mathrm{C}-\mathrm{NMR}$ (125 MHz, DMSO- $\left.d_{6}\right)$ : $42.11\left(\mathrm{CH}_{2}\right), 42.67\left(\mathrm{CH}_{2}\right)$, $59.32(\mathrm{CH}), 112.88(\mathrm{CH}), 115.72(\mathrm{CH}), 127.95(2 \mathrm{CH}), 129.18$ (2CH), $132.56(\mathrm{C}), 140.56(\mathrm{C}), 145.98(\mathrm{CH}), 146.47(\mathrm{C}), 147.54$ (C), $163.78(\mathrm{C})$.

For $\mathrm{C}_{15} \mathrm{H}_{12} \mathrm{Cl}_{2} \mathrm{~N}_{2} \mathrm{O}_{2}$ calculated: $55.75 \% \mathrm{C}, 3.74 \% \mathrm{H}, 8.67 \% \mathrm{~N}$; found: $55.83 \% \mathrm{C}, 3.76 \% \mathrm{H}, 8.61 \% \mathrm{~N}$.

MS (FAB) $[\mathrm{M}+1]^{+}: \mathrm{m} / \mathrm{z} 323$

\section{1-(Chloroacetyl)-3-(2-furanyl)-5-(4-fluorophenyl)-2- pyrazoline (5f)}

IR (KBr) vmax $\left(\mathrm{cm}^{-1}\right): 1683(\mathrm{C}=\mathrm{O}), 1564,1510,1463(\mathrm{C}=\mathrm{N}$ and $\mathrm{C}=\mathrm{C})$.

${ }^{1} \mathrm{H}-\mathrm{NMR}\left(500 \mathrm{MHz}, \delta \mathrm{ppm}, \mathrm{DMSO}-d_{6}\right): 3.08(1 \mathrm{H}, \mathrm{dd}, J=18.08$, $4.59 \mathrm{~Hz}), 3.80(1 \mathrm{H}, \mathrm{dd}, J=18.06,11.71 \mathrm{~Hz}), 4.61(1 \mathrm{H}, \mathrm{d}, J=13.98$ $\mathrm{Hz}), 4.72(1 \mathrm{H}, \mathrm{d}, J=13.92 \mathrm{~Hz}), 5.57(1 \mathrm{H}, \mathrm{dd}, J=11.75,4.58 \mathrm{~Hz})$, $6.68(1 \mathrm{H}, \mathrm{t}, J=3.08,1.52 \mathrm{~Hz}), 7.05(1 \mathrm{H}, \mathrm{d}, J=3.50 \mathrm{~Hz}), 7.18(2 \mathrm{H}$, $\mathrm{d}, J=8.50 \mathrm{~Hz}), 7.27(2 \mathrm{H}, \mathrm{d}, J=8.49 \mathrm{~Hz}), 7.93(1 \mathrm{H}, \mathrm{s})$.

For $\mathrm{C}_{15} \mathrm{H}_{12} \mathrm{ClFN}_{2} \mathrm{O}_{2}$ calculated: $58.74 \%$ C, $3.94 \% \mathrm{H}, 9.13 \% \mathrm{~N}$; found: $58.65 \% \mathrm{C}, 3.87 \% \mathrm{H}, 9.21 \% \mathrm{~N}$.

MS (FAB) $[\mathrm{M}+1]^{+}: \mathrm{m} / \mathrm{z} 307$

1-(Chloroacetyl)-3-(2-thienyl)-5-(3,4methylenedioxyphenyl)-2-pyrazoline (5g)

IR (KBr) vmax (cm-1): 1679 (C=O), 1564, 1500, 1452, 1415 (C=N and $\mathrm{C}=\mathrm{C})$.

${ }^{1} \mathrm{H}-\mathrm{NMR}\left(500 \mathrm{MHz}, \delta \mathrm{ppm}, \mathrm{DMSO}-d_{6}\right): 3.19(1 \mathrm{H}, \mathrm{dd}, J=18.02$, $4.58 \mathrm{~Hz}), 3.86(1 \mathrm{H}, \mathrm{dd}, J=18.03,11.57 \mathrm{~Hz}), 4.60(1 \mathrm{H}, \mathrm{d}, J=13.84$ $\mathrm{Hz}), 4.71(1 \mathrm{H}, \mathrm{d}, J=13.86 \mathrm{~Hz}), 5.52(1 \mathrm{H}, \mathrm{dd}, J=11.59,4.57 \mathrm{~Hz})$, $6.04(2 \mathrm{H}, \mathrm{s}), 6.71(1 \mathrm{H}, \mathrm{dd}, J=8.05,1.50 \mathrm{~Hz}), 6.77(1 \mathrm{H}, \mathrm{d}, J=1.52$ $\mathrm{Hz}), 6.87(1 \mathrm{H}, \mathrm{d}, J=8.04 \mathrm{~Hz}), 7.17(1 \mathrm{H}, \mathrm{t}, J=5.02,3.50 \mathrm{~Hz}), 7.50$ $(1 \mathrm{H}, \mathrm{d}, J=3.52 \mathrm{~Hz}), 7.79(1 \mathrm{H}, \mathrm{d}, J=5.01 \mathrm{~Hz})$.

${ }^{13} \mathrm{C}-\mathrm{NMR}\left(125 \mathrm{MHz}, \mathrm{DMSO}-d_{6}\right): 42.86\left(\mathrm{CH}_{2}\right), 43.20\left(\mathrm{CH}_{2}\right)$, $60.27(\mathrm{CH}), 101.52\left(\mathrm{CH}_{2}\right), 106.51(\mathrm{CH}), 108.80(\mathrm{CH}), 119.32$ $(\mathrm{CH}), 128.60(\mathrm{CH}), 130.65(\mathrm{CH}), 131.58(\mathrm{CH}), 134.07(\mathrm{C}), 135.78$ (C), 147.00 (C), 148.01 (C), 152.04 (C), 163.33 (C).

For $\mathrm{C}_{16} \mathrm{H}_{13} \mathrm{ClN}_{2} \mathrm{O}_{3} \mathrm{~S}$ calculated: $55.10 \% \mathrm{C}, 3.76 \% \mathrm{H}, 8.03 \% \mathrm{~N}$; found: $55.18 \% \mathrm{C}, 3.73 \% \mathrm{H}, 8.11 \% \mathrm{~N}$.

MS (FAB) $[\mathrm{M}+1]^{+}: \mathrm{m} / \mathrm{z} 349$

1-(Chloroacetyl)-3-(2-thienyl)-5-(4-bromophenyl)-2-pyrazoline (5h)

IR (KBr) vmax $\left(\mathrm{cm}^{-1}\right): 1666$ (C=O), 1558, 1448, 1425, $1413(\mathrm{C}=\mathrm{N}$ and $\mathrm{C}=\mathrm{C}$ ).

${ }^{1} \mathrm{H}-\mathrm{NMR}\left(500 \mathrm{MHz}, \delta\right.$ ppm, DMSO- $\left.d_{6}\right): 3.22(1 \mathrm{H}, \mathrm{dd}, J=18.05$, $4.57 \mathrm{~Hz}), 3.91(1 \mathrm{H}, \mathrm{dd}, J=18.03,11.55 \mathrm{~Hz}), 4.61(1 \mathrm{H}, \mathrm{d}, J=13.86$
$\mathrm{Hz}), 4.71(1 \mathrm{H}, \mathrm{d}, J=13.89 \mathrm{~Hz}), 5.58(1 \mathrm{H}, \mathrm{dd}, J=11.56,4.54 \mathrm{~Hz})$, $7.16(1 \mathrm{H}, \mathrm{t}, J=3.51,1.04 \mathrm{~Hz}), 7.20(2 \mathrm{H}, \mathrm{d}, J=8.50 \mathrm{~Hz}), 7.50(1 \mathrm{H}$, $\mathrm{d}, J=3.50 \mathrm{~Hz}), 7.55(2 \mathrm{H}, \mathrm{d}, J=8.51 \mathrm{~Hz}), 7.80(1 \mathrm{H}, \mathrm{d}, J=5.03 \mathrm{~Hz})$.

${ }^{13} \mathrm{C}-\mathrm{NMR}\left(125 \mathrm{MHz}, \mathrm{DMSO}-d_{6}\right): 42.76\left(\mathrm{CH}_{2}\right), 42.97\left(\mathrm{CH}_{2}\right)$, $59.97(\mathrm{CH}), 121.01(\mathrm{C}), 128.41(2 \mathrm{CH}), 128.62(\mathrm{CH}), 130.32(\mathrm{CH})$, $131.71(\mathrm{CH}), 132.10(2 \mathrm{CH}), 133.90(\mathrm{C}), 141.23(\mathrm{C}), 152.08(\mathrm{C})$, $163.44(\mathrm{C})$.

For $\mathrm{C}_{15} \mathrm{H}_{12} \mathrm{BrClN}_{2} \mathrm{OS}$ calculated: $46.96 \%$ C, $3.15 \% \mathrm{H}, 7.30 \% \mathrm{~N}$; found: $46.93 \% \mathrm{C}, 3.19 \% \mathrm{H}, 7.23 \% \mathrm{~N}$.

MS (FAB) $[\mathrm{M}+1]^{+}: \mathrm{m} / \mathrm{z} 384$

\section{Microbiology \\ Antimicrobial activity}

The in vitro antimicrobial activities of the compounds (5a-h) were tested using the microbroth dilution method (18). Tested microorganism strains were Staphylococcus aureus (NRRL B-767), Listeria monocytogenes (ATCC-7644), Escherichia coli (ATCC-25922), Pseudomonas aeruginosa (ATCC-254992), Micrococcus luteus (NRLL B-4375), Bacillus subtilis (NRS-744) and Candida albicans (ATCC-22019). Microbroth dilution-susceptibility assay was used for antimicrobial evaluation of the compounds. Stock solutions of the samples were prepared in dimethyl sulfoxide. Dilution series using sterile distilled water were prepared from $4 \mathrm{mg} / \mathrm{mL}$ to $0.0039 \mathrm{mg} / \mathrm{mL}$ in microtest tubes that were transferred to 96 -well microtiter plates. Overnight-grown bacterial and C. albicans suspensions in doublestrength Mueller-Hinton broth were standardized to $10^{8} \mathrm{CFU} /$ $\mathrm{mL}$ using McFarland No: 0.5 standard solutions. Hundred microliter of each microorganism suspension was then added into the wells. The last well-chain without a microorganism was used as a negative control. Sterile distilled water and the medium served as a positive growth control. After incubation at $37{ }^{\circ} \mathrm{C}$ for $18-24 \mathrm{~h}$, antimicrobial activity was detected by spraying of $0.5 \%$ TTC (triphenyltetrazolium chloride, Merck) aqueous solution. Minimum Inhibitory Concentration (MIC) was defined as the lowest concentration of compounds that inhibited visible growth, as indicated by the TTC staining. Streptomycin was used as standard antibacterial agent, whereas ketoconazole was used as an antifungal agent. Observed data on the antimicrobial activity of the compounds and control drugs are given in Table 2.

\section{RESULTS AND DISCUSSION}

Initially, the chalcones (1-(2-furanyl/thienyl)-3-aryl-2-propen1-ones) (3a-h) were synthesized by literature methods as described $(16,17)$ and treated with hydrazine hydrate $(80 \%)$ to obtain 5-aryl-3-(2-furanyl/thienyl)-2-pyrazolines (4a-h) (Scheme 1).

The desired 1-(chloroacetyl)-3-(2-furanyl/thienyl)-5-aryl2-pyrazolines (5a-h) were synthesized via the nucleophilic acyl substitution reactions of 5-aryl-3-(2-furanyl/thienyl)2-pyrazolines with chloroacetyl chloride in the presence of triethylamine. These reactions are summarized in Scheme 1 and some properties of the compounds are given in Table 1.

The structures of the compounds (5a-h) were confirmed by IR, ${ }^{1} \mathrm{H}-\mathrm{NMR},{ }^{13} \mathrm{C}-\mathrm{NMR}$ and $\mathrm{FAB}^{+}-\mathrm{MS}$ spectral data and Elemental analyses. 
<smiles></smiles>

1<smiles>[R]c1ccc(C=O)cc1</smiles>

2<smiles>[R]c1ccc(C=CC(=O)c2ccc[X]2[H])cc1</smiles>

3a-h<smiles></smiles>

5a-h

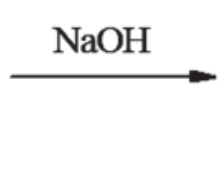

$\downarrow \mathrm{NH}_{2} \mathrm{NH}_{2} \cdot \mathrm{H}_{2} \mathrm{O}$

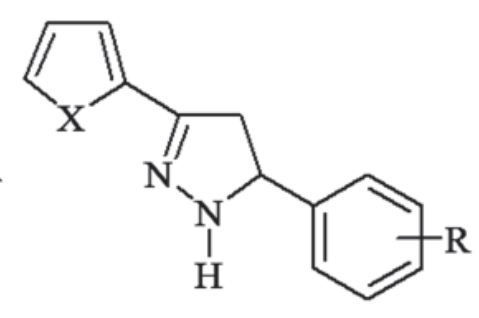

4a-h

SCHEME 1. The synthetic protocol of the compounds (5a-h)

In the IR spectra of all compounds (5a-h), all derivatives have a strong, characteristic band in the region $1658-1683 \mathrm{~cm}^{-1}$ due to the amide $\mathrm{C}=\mathrm{O}$ stretching vibration. The bands due to the $\mathrm{C}=\mathrm{C}$ and $\mathrm{C}=\mathrm{N}$ stretching vibrations are observed in the region $1610-1413 \mathrm{~cm}^{-1}$.

The ${ }^{1} \mathrm{H}-\mathrm{NMR}$ spectral data were also consistent with the assigned structures. In the $500 \mathrm{MHz}{ }^{1} \mathrm{H}-\mathrm{NMR}$ spectra of compounds, the $\mathrm{CH}_{2}$ protons of the pyrazoline ring resonated as a pair of doublets of doublets at $\delta 3.04-3.22$ ppm, 3.78-3.91 ppm. The $\mathrm{CH}$ proton appeared as doublet of doublets at $\delta 5.48-5.58$ ppm due to vicinal coupling with the two magnetically nonequivalent protons of the methylene group at position 4 of the pyrazoline ring $\left(J_{\mathrm{AM}}=18.01-18.08 \mathrm{~Hz}, J_{\mathrm{AX}}=4.51-4.74 \mathrm{~Hz}, J_{\mathrm{MX}}\right.$ $=11.48-11.75 \mathrm{~Hz})$. The $\mathrm{CH}_{2}$ protons of acetyl (5a-h) are observed in the region $4.57-4.73 \mathrm{ppm}$ as double doublets $(J=$ 13.73-13.98 Hz, $J=13.71-13.92 \mathrm{~Hz}$ ). This geminal coupling result from steric structure of the compounds. These geminal protons are observed as double doublet due to possible two different conformations since rigid protons were occurred $(19,20)$. All the other aromatic and aliphatic protons were observed at expected regions.

In the ${ }^{13} \mathrm{C}-\mathrm{NMR}$ spectra of the compounds, the signal due to the carbonyl carbon appears at 163.28-163.78 ppm. ${ }^{13} \mathrm{C}-\mathrm{NMR}$ chemical shift values of the carbon atoms at $42.11-42.86 \mathrm{ppm}$ (C-4), 59.32-60.27 ppm (C-5), and about 147.39-152.62 ppm (C3) corroborate the 2-pyrazoline character deduced from the
TABLE 1. Some properties of the synthesized compounds (5a-h)

\begin{tabular}{ccccccc}
\hline Compound & $\mathbf{R}$ & $\mathbf{X}$ & $\begin{array}{c}\text { Yield } \\
(\%)\end{array}$ & $\begin{array}{c}\text { M.p. } \\
\text { ('C) }\end{array}$ & $\begin{array}{c}\text { Molecular } \\
\text { formula }\end{array}$ & $\begin{array}{c}\text { Molecular } \\
\text { weight }\end{array}$ \\
\hline $5 \mathrm{a}$ & $\mathrm{CH}_{3}$ & $\mathrm{O}$ & 61 & 81 & $\mathrm{C}_{16} \mathrm{H}_{15} \mathrm{CIN}_{2} \mathrm{O}_{2}$ & 302 \\
$5 \mathrm{~b}$ & $\mathrm{O}-\mathrm{CH}_{2}-\mathrm{O}$ & $\mathrm{O}$ & 67 & 104 & $\mathrm{C}_{16} \mathrm{H}_{13} \mathrm{CIN}_{2} \mathrm{O}_{4}$ & 332 \\
$5 \mathrm{c}$ & $\mathrm{OCH}_{3}$ & $\mathrm{O}$ & 63 & 150 & $\mathrm{C}_{16} \mathrm{H}_{15} \mathrm{ClN}_{2} \mathrm{O}_{3}$ & 318 \\
$5 \mathrm{~d}$ & $\mathrm{Br}$ & $\mathrm{O}$ & 58 & 185 & $\mathrm{C}_{15} \mathrm{H}_{12} \mathrm{BrClN}_{2} \mathrm{O}_{2}$ & 367 \\
$5 \mathrm{e}$ & $\mathrm{Cl}$ & $\mathrm{O}$ & 70 & 125 & $\mathrm{C}_{15} \mathrm{H}_{12} \mathrm{Cl}_{2} \mathrm{~N}_{2} \mathrm{O}_{2}$ & 322 \\
$5 f$ & $\mathrm{~F}$ & $\mathrm{O}$ & 62 & 149 & $\mathrm{C}_{15} \mathrm{H}_{12} \mathrm{ClFN}_{2} \mathrm{O}_{2}$ & 306 \\
$5 \mathrm{~g}$ & $\mathrm{O}-\mathrm{CH}_{2}-\mathrm{O}$ & $\mathrm{S}$ & 70 & 137 & $\mathrm{C}_{16} \mathrm{H}_{13} \mathrm{CIN}_{2} \mathrm{O}_{3} \mathrm{~S}$ & 348 \\
$5 \mathrm{~h}$ & $\mathrm{Br}$ & $\mathrm{S}$ & 60 & 146 & $\mathrm{C}_{15} \mathrm{H}_{12} \mathrm{BrClN}_{2} \mathrm{OS}$ & 383
\end{tabular}

${ }^{1} \mathrm{H}-\mathrm{NMR}$ data. The other aromatic and aliphatic carbons were observed at expected regions.

In the mass spectra of all compounds (5a-h), the $M+1$ peak is observed. All compounds gave satisfactory elemental analyses.

The antimicrobial activity of the compounds was studied with seven pathogenic microorganisms. Streptomycin and ketoconazole were used as reference drugs. The results are summarized in Table 2.

All compounds (5a-h) exhibited the highest antibacterial activity against $P$. aeruginosa with a MIC value of $250 \mu \mathrm{g} / \mathrm{mL}$ among the tested bacteria. The results clearly indicated that the func- 
TABLE 2. Antimicrobial activities of the compounds (5a-h) $(\mu \mathrm{g} / \mathrm{mL})$

\begin{tabular}{cccccccc}
\hline & A & B & C & D & E & $\mathbf{F}$ & G \\
\hline $5 a$ & 125 & 250 & 250 & 250 & 125 & 125 & 125 \\
$5 b$ & 250 & 250 & 250 & 250 & 250 & 250 & 125 \\
$5 c$ & 250 & 250 & 250 & 250 & 250 & 250 & 125 \\
$5 d$ & 250 & 250 & 250 & 250 & 125 & 250 & 125 \\
$5 \mathrm{f}$ & 250 & 500 & 250 & 250 & 250 & 250 & 250 \\
$5 f$ & 250 & 250 & 250 & 250 & 250 & 250 & 125 \\
$5 \mathrm{~g}$ & 250 & 250 & 250 & 250 & 125 & 250 & 250 \\
$5 \mathrm{fh}$ & 250 & 250 & 250 & 250 & 250 & 250 & 125 \\
Streptomycin & 31.25 & 7.81 & 31.25 & 125 & 15.62 & 15.62 & - \\
Ketoconazole & - & - & - & - & - & - & 250 \\
\hline A: S. aureus (NRRL B-767), B: L. monocytogenes (ATCC-7644), C: E. coli (ATCC- \\
25922), D: P. aeruginosa (ATCC-254992), E: M. luteus (NRLL B-4375), F: B. subtilis \\
(NRS-744), G: C. albicans (ATCC-22019)
\end{tabular}

tional groups on 2-pyrazoline ring did not have a considerable influence on the antibacterial activity against $P$. aeruginosa.

Compound 5a exhibited antibacterial activity against $S$. aureus with a MIC value of $125 \mu \mathrm{g} / \mathrm{mL}$. Among all compounds (5a-h), compound $\mathbf{5 a}$ also displayed the highest antibacterial activity against $B$. subtilis. Compounds $\mathbf{5 a}, \mathbf{5} \mathbf{d}$ and $\mathbf{5 g}$ showed moderate activity M. luteus with a MIC value of $125 \mu \mathrm{g} / \mathrm{mL}$. All compounds (5a-h) displayed the same antibacterial activity against E. coli with a MIC value of $250 \mu \mathrm{g} / \mathrm{mL}$.
All compounds except compounds $5 \mathbf{e}$ and $5 \mathrm{~g}$ are more effective than ketoconazole against $C$. albicans, whereas compounds $5 \mathrm{e}, 5 \mathrm{~g}$ and ketoconazole showed the same level of antifungal activity against $C$. albicans.

\section{CONCLUSION}

In conclusion, the synthesis of 2-pyrazoline derivatives was described and their in vitro antimicrobial effects of these compounds on various pathogenic bacteria and C. albicans were evaluated. The biological results indicate that $P$. aeruginosa and $C$. albicans are more susceptible to the synthesized compounds.

Considering all the results obtained from antimicrobial screen, in comparison with reference agents, it can be concluded that compound $\mathbf{5 a}$ is the most effective compound in the screen.

Based on eight compounds evaluated, it appears that 4-methyl substitution on the phenyl ring (5a) has made a good contribution to the antimicrobial activity in this series of pyrazolinehaloacetamide combination.

\section{ACKNOWLEDGEMENTS}

The author would like to thank Anadolu University Medicinal Plants, Drugs and Scientific Research Center (AUBIBAM) for spectroscopic analyses and biological activity tests.

\section{Amit parçası taşıyan bazı pirazolin türevlerinin sentezi ve antimikrobiyal etkileri}

ÖZET: Bu çalışmada, bir seri pirazolin türevi sentezlendi. Bileşiklerin kimyasal yapıları IR, ${ }^{1} \mathrm{H}-\mathrm{NMR},{ }^{13} \mathrm{C}-\mathrm{NMR}$, FAB-MS spektral verileri ve elemental analiz ile aydınlatıldı. Sentezlenen bileşikler antimikrobiyal etkileri tarandı. Bütün bileşikler en yüksek antibakteriyel etkiyi $P$. aeruginosa'ya karşı gösterdiler. 1-(Kloroasetil)-3-(2-furanil)-5-(4-klorofenil)-2pirazolin ve 1-(kloroasetil)-3-(2-tiyenil)-5-(3,4-metilendioksifenil)-2-pirazolin bileşikleri hariç bütün bileşikler $C$. albicans'a karşı ketokonazolden daha etkili iken, 1-(kloroasetil)-3-(2-furanil)-5-(4-klorofenil)-2-pirazolin ve 1-(kloroasetil)-3-(2-tiyenil)-5-(3,4-metilendioksifenil)-2-pirazolin bileşikleri C. albicans'a karşı ketokonazol ile aynı düzeyde antifungal etki gösterdiler.

ANAHTAR KELIMELER: pirazolin, amit, furan, tiyofen, antimikrobiyal etki

\section{REFERENCES}

1. Alanis AJ. Resistance to antibiotics: Are we in the postantibiotic era?. Arch Med Res 2005; 36: 697-705.

2. Ghannoum MA, Louis BR. Antifungal agents: mode of action, mechanisms of resistance and correlation of these mechanisms with bacterial resistance. Clin Microbiol Rev 1999; 12: 501-17.

3. Palkar M, Noolvi M, Sankangoud R, Maddi V, Gadad A, Nargund LVG. Synthesis and antibacterial activity of a novel series of 2,3-diaryl-substituted-imidazo(2,1-b)benzothiazole derivatives. Arch Pharm Chem Life Sci 2010; 343: 353-9.

4. Lemke TL, Williams DA. Foye's Principles of Medicinal Chemistry. Lippincott Williams\&Wilkins, Baltimore and Philadelphia. 2008.
5. Papich MG, Riviere JE. Veterinary pharmacology and therapeutics. Wiley-Blackwell, Iowa. 2009.

6. Ciapetti P, Giethlen B. The Practice of Medicinal Chemistry. Academic Press, Burlington. 2008.

7. Lima LM, Barreiro EJ. Bioisosterism: A useful strategy for molecular modification and drug design. Curr Med Chem 2005; 12: 23-49.

8. Patani GA, LaVoie EJ. Bioisosterism: A rational approach in drug design. Chem Rev 1996; 96: 3147-76.

9. Narang R, Narasimhan B, Sharma S. A review on biological activities and chemical synthesis of hydrazide derivatives. Curr Med Chem 2012; 19: 569-612.

10. Suresh K, Sandhya B, Sushma D, Rajiv K, Himanshu G. Biological activities of pyrazoline derivatives - A recent development. Recent Patents Anti-Infect Drug Discov 2009; 4: 154-63. 
11. Kaplancıklı ZA, Turan-Zitouni G, Özdemir A, Revial G, Güven K. Synthesis and antimicrobial activity of some thiazolyl-pyrazoline derivatives. Phosphorus Sulfur Silicon Relat Elem 2007; 182: 749-64.

12. Özdemir A, Turan-Zitouni G, Kaplancıklı ZA, Revial G, Güven K. Synthesis and antimicrobial activity of 1-(4-aryl-2-thiazolyl)-3-(2-thienyl)-5-aryl-2-pyrazoline derivatives. Eur J Med Chem 2007; 42: 403-9.

13. Özdemir A, Turan-Zitouni G, Kaplancıklı ZA, Revial G, Demirci F, Iscan G. Preparation of some pyrazoline derivatives and evaluation of their antifungal activities. J Enzym Inhib Med Chem 2010; 25: 565-71.

14. Turan-Zitouni G, Özdemir A, Güven K. Synthesis of some 1-[(N,Ndisubstituted thiocarbamoylthio)acetyl]3-(2-thienyl)-5-aryl-2-pyrazoline derivatives and investigation of their antibacterial and antifungal activities. Arch Pharm Pharm Med Chem 2005; 338: 96-104.

15. Turan-Zitouni G, Özdemir A, Kaplancıklı ZA. Synthesis and antimicrobial activities of some 1-[(N,N-disubstitutedthiocarbamoylthio)acetyl]-3,5-diaryl-2-pyrazolines. Phosphorus Sulfur Silicon Relat Elem 2005; 180: 2717-24.
16. Kabli RA, Khalaf AA, Zimaity MT, Khalil AM, Kaddah AM, Al-Rifaie HA. Synthesis of a new series of furyl and thienyl substituted pyrazolines starting with furyl and thienyl chalcones. J Indian Chem Soc 1991; 68: 47-51.

17. Khalaf AA, Kabli RA, Zimaity MT, Khalil AM, Kaddah AM, Al-Rifaie, HA. N-Derivatization of some 3-(2-furyl)and 3-(2-thienyl)-5-aryl-2-pyrazolines. Indian J Chem Sect B 1993; 32: 1125-9.

18. Winn WC, Allen SD, Janda WM, Koneman EW, Procop GW, Schreckenberger PC, Woods GL. Koneman's color atlas and textbook of diagnostic microbiology. Lippincott Williams\&Wilkins, Baltimore and Philadelphia. 2006.

19. Ledneczki I, Forgo P, Kiss JT, Molnár Á, Pálinkó I. Conformational behaviour of acetamide derivatives studied by NMR spectroscopic and computational methods. J Mol Struct 2007; 834: 349-54.

20. Vijayabaskar V, Perumal S, Selvaraj S, Lycka A, Murugan $R$, Balasubramanian $M$. Synthesis and multinuclear NMR study of (E)-s-trans-1-acetyl-5-aryl-3-styryl2-pyrazolines. Magn Reson Chem 1999; 37: 133-9. 